\title{
Quality assurance for dynamic tumor tracking using the Vero4DRT system
}

\author{
Hideharu Miura1, Shuichi Ozawa',2, Shintaro Tsuda1, Masahiro Hayata1, \\ Kiyoshi Yamada1, Yasushi Nagata, ${ }^{1,2}$
}

\author{
${ }^{1}$ Hiroshima High-Precision Radiotherapy Cancer Center, Hiroshima, Japan \\ ${ }^{2}$ Department of Radiation Oncology, Institute of Biomedical \& Health Science, Hiroshima University, Hiroshima, Japan
}

Received November 18, 2015; Revised December 27, 2015; Accepted January 20, 2016; Published Online February 06, 2016

\section{Original Article}

\begin{abstract}
Purpose: We perform quality assurance $(\mathrm{Q} A)$ for indirect dynamic tumor tracking (DTT) using four-dimensional radiation therapy (the Vero4DRT ${ }^{\text {TM }}$ system). Methods: A single photon beam was set with a $40 \times 40 \mathrm{~mm}^{2}$ field size at a gantry angle of zero degrees and a low monitor unit setting of 200. Doses were measured using a $0.016 \mathrm{~cm}^{3}$ ionization chamber inserted in a phantom under stationary, DTT, and non-DTT conditions for sinusoidal (peak-to-peak) amplitude $[A]$ and breathing period [T] (20 mm, $2 \mathrm{~s} ; 20 \mathrm{~mm}, 4 \mathrm{~s}$; and $40 \mathrm{~mm}, 4 \mathrm{~s})$. The stationary condition was measured for comparison. Dose profiles were measured using Gafchromic EBT3 films in the phantom under the same conditions. Results: For chamber measurement, the relative doses were as follows: 0.99 with non-DTT and 1.00 with DTT at $A=20 \mathrm{~mm}$ and $T=2 \mathrm{~s} ; 0.99$ with non-DTT and 1.00 with DTT at $A=20 \mathrm{~mm}$ and $T=4 \mathrm{~s}$; and 0.84 with non-DTT and 1.00 with DTT at $A=40 \mathrm{~mm}$ and $T=4 \mathrm{~s}$. For film measurement, the spatial distances between the $90 \%$ dose of the dose profiles were as follows: $6.53 \mathrm{~mm}$ for non-DTT and $0.40 \mathrm{~mm}$ for DTT at $A=20 \mathrm{~mm}$ and $T=2 \mathrm{~s} ; 6.33 \mathrm{~mm}$ for non-DTT and $0.15 \mathrm{~mm}$ for DTT at $A=20 \mathrm{~mm}$ and $T=4 \mathrm{~s}$; and $10.61 \mathrm{~mm}$ for non-DTT and $0.17 \mathrm{~mm}$ with DTT at $A=40 \mathrm{~mm}$ and $T=4 \mathrm{~s}$. Conclusion: Our results showed high dosimetric and geometric accuracy for DTT using four-dimensional modeling and marked reduction of the blurring effects on dose distribution. We recommend the use of a QA procedure for DTT performed using the Vero4DRT ${ }^{\mathrm{TM}}$ system.
\end{abstract}

Keywords: Vero4DRT; Dynamic Tumor Tracking; Quality Assurance; 4D modeling

\section{Introduction}

Through breathing management, stereotactic body radiation therapy (SBRT) can deliver increased doses of radiation to tumors while decreasing the dose delivered to normal tissue. ${ }^{1-3}$ Several methods for controlling respiratory motion have been reported, including breath holding 4 , respiratory gating 5 , and dynamic tumor tracking (DTT).$^{6-8}$ Breath holding is more difficult for patients who cannot repeatedly hold their breath over the delivery time. Respiratory gating needs a longer treatment time to deliver the dose within a particular portion of the patient's breathing cycle. Therefore, neither of these methods is ideal. In contrast, DTT is an innovative technique that does not require either a longer treatment time or the burden of breath holding. ${ }^{6-8}$

DTT can be categorized into direct and indirect methods. The Vero4DRT ${ }^{\text {TM }}$ system is a four-dimensional (4D) radiation therapy that uses indirect DTT approaches with external signals in consideration of the risks associated with the increased radiation dose delivered by Kilovoltage $(\mathrm{kV}) \mathrm{X}$-ray imaging. The DTT techniques of the Vero4DRT ${ }^{\mathrm{TM}}$ system require synchronization of the gimbals swing with the patient's respiratory cycle, which is based on 4D modeling. Several investigators have reported the tracking accuracy of DTT in the Vero4DRT $^{\text {TM }}$ system using chamber and film measurement. ${ }^{6-7}$ The American Association of Physicists in Medicine (AAPM) Task Group (TG) 76 reported on the quality assurance $(\mathrm{QA})$ of all respiratory techniques. ${ }^{9}$ The AAPM-TG 142 also recommended monthly and annual tolerance and function checks for respiratory gating. ${ }^{10}$ However, the tolerance values have not been stated because DTT technique was rarely used in 2009.

Corresponding author: Hideharu Miura; Hiroshima High-Precision Radiotherapy Cancer Center, Hiroshima, Japan.

Cite this article as: Miura H, Ozawa S, Tsuda S, Hayata M, Yamada K, Nagata Y. Quality assurance for dynamic tumor tracking using the Vero4DRT system. Int J Cancer Ther Oncol. 2016; 4(1):4112. DOI: 10.14319/ijcto.41.12 
In this study, we assessed the chamber and film measurements to confirm tracking accuracy by a comparison with the non-DTT results. We also calculated the 4D-modeling error between the predicted and detected target motions. Finally, we compared whether the 4D-modeling-error and measurement results were correlated.

\section{Methods and Materials}

\subsection{Vero4DRT system}

We perform QA for indirect DTT using the Vero4DRT ${ }^{\mathrm{TM}}$ system in our institution. The Vero4DRT ${ }^{\mathrm{TM}}$ system (MHI-TM2000; Mitsubishi Heavy Industries, Ltd., Tokyo, Japan, and Brainlab, Feldkichen, Germany) is described elsewhere. ${ }^{6-8}$ Gimbaled X-ray head mounted on an O-ring gantry with a C-band klystron, a system-specific fixed jaw, and a multileaf collimator (MLC). The gimbaled X-ray head can swing along two orthogonal axes up to $\pm 2.5^{\circ}$ (swings the beam up to $\pm 41.9 \mathrm{~mm}$ in each direction on the isocenter plane), allowing pan and tilt motion of the linac. The Vero4DRT ${ }^{\mathrm{TM}}$ system uses a fully integrated target-positioning concept, an ExacTrac system version 3.5.3 (BrainLAB AG, Feldkirchen, Germany) automated infrared (IR) camera mounted on the ceiling of the treatment room, and two orthogonal $\mathrm{kV} \mathrm{X}$-ray imaging systems attached to the 0-ring at $45^{\circ}$ from the MV beam axis. The $\mathrm{kV}$ X-ray imaging systems acquire cone-beam computed tomography (CBCT) data using the 0 -ring rotation.

\subsection{Experimental procedure}

In Figure 1, the entire phantom system can be seen. The programmable respiratory motion table (CIRS. Inc., Norfolk, VA, USA) was used to simulate breathinginduced organ motion. The dynamic phantom was capable of motion based on an arbitrary input function, and the motion table had one table that moved in the horizontal direction and one that moved in the vertical direction (the motions of these tables were
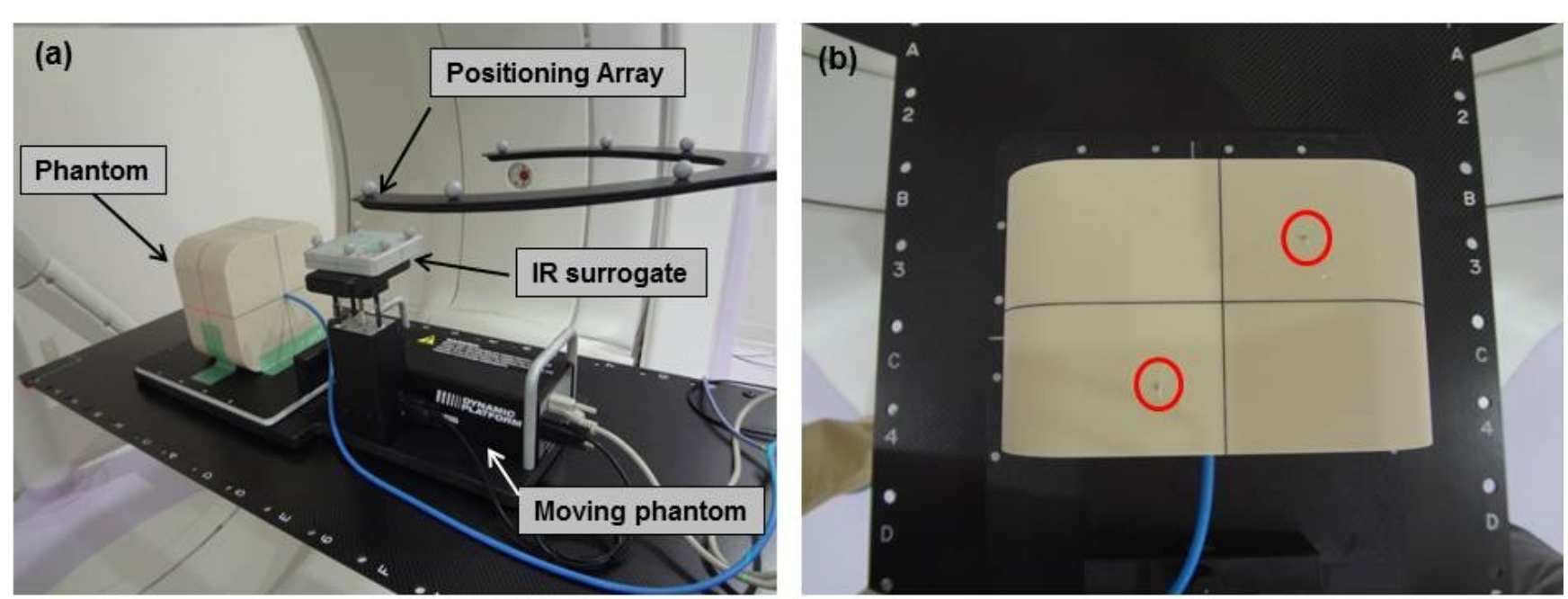

Figure 1: (a) Experimental setup for a single field. The phantom was placed on the motion table. Then, the chamber and film

were inserted in the phantoms. (b) Two iron markers were attached to the phantom's surface (red circle). synchronized). We used iron markers as substitutes for gold ones. Two iron markers, each with a diameter of 2.0 $\mathrm{mm}$, were attached to the phantom surfaces; at least two markers were required for establishing 4D modeling function. The center of the phantom was set at the isocenter of the motion table that moved in the horizontal direction. An IR phantom was positioned on the vertical direction motion table as a surrogate signal.

4D modeling with the Vero4DRT ${ }^{\mathrm{TM}}$ system is described elsewhere.11-12 Briefly, two iron markers on the cube phantom and the IR markers on the vertical table are simultaneously acquired to calculate the 4D modeling function. A pair of orthogonal $\mathrm{kV} \mathrm{X}$-rays at gantry angles of $0^{\circ}$ and $90^{\circ}$ acquired the positions of the iron markers every 320 or $640 \mathrm{~ms}$. The sampling interval of the $\mathrm{kV}$ $\mathrm{X}$-ray images automatically changed to $640 \mathrm{~ms}$ when the velocity of the IR marker motion decreased. The acquisition times ranged from 20 to $40 \mathrm{~s}$. The center of the two markers during motion was defined as the detected target position. The motion of the surrogate was acquired from the IR markers attached on the phantom monitored by the IR camera on the ceiling of the treatment room every $16.7 \mathrm{~ms}$. The 4D modeling function was a quadratic function of the IR marker position and velocity,

$$
P_{\text {predict }}=a P_{I R}^{2}+b P_{I R}+c+d v_{I R}^{2}+e v_{I R} \text {, }
$$

where, $P_{\text {predict }}$ is the predicted target position, $P_{I R}$ is the IR marker positions and $v_{I R}$ is the vertical velocity of the IR markers. Parameters $a, b, c, d$, and $e$ were optimized by minimizing the residual errors between $P$ predict and the predicted target position for each IR marker. After analyzing the $4 \mathrm{D}$ motion data of the target and IR marker motions, the 4D modeling function was calculated by the ExacTrac system. The target position calculated by the 4D modeling function was defined as the predicted target position. 
The iPlan RT Dose ${ }^{\mathrm{TM}}$ treatment planning system version 4.5.3 (BrainLAB, Feldkirchen, Germany) was used for plan design. A single photon beam was set with a $40 \times$ $40-\mathrm{mm}^{2}$ field size at a gantry angle of zero degrees and a low monitor unit setting of 200 as the reference in the DTT accuracy comparisons. Sinusoidal motion sequences were produced in the dynamic phantom, using different amplitudes and breathing period. To investigate correlation between the 4D modeling error and the tracking accuracy, we performed the following peak-to-peak amplitudes $(A)$ and breathing period $(T)$ assessments: 1D sinusoidal patterns $(A, T)=(20 \mathrm{~mm}, 2$ $\mathrm{s}),(20 \mathrm{~mm}, 4 \mathrm{~s})$, and (40 mm, $4 \mathrm{~s})$ and a volunteer's respiratory pattern (20 $\mathrm{mm}$, average of $4 \mathrm{~s}$ ) in the cranio-caudal (CC) direction. IR marker motion was fixed at a peak-to-peak amplitude of $20 \mathrm{~mm}$ and the breathing period was synchronized with the target motion. Irradiation fields were then delivered under three conditions: with the phantom moving and DTT based on the motion of the phantom, with the phantom moving but with non-DTT, and with a stationary phantom (for comparison).

Dose outputs were measured using a $0.016 \mathrm{~cm}^{3}$ ionization chamber (PTW31016 pinpoint chamber; PTW, GmbH, Freiburg, Germany) in the center of phantom. Dose values were relative to the dose measured for a static beam with stationary phantom.

Gafchromic EBT3 films (International Specialty Products Corporation, Wayne, NJ, USA) were inserted in the phantom and irradiated under the same conditions as those for the chamber measurement. An Epson Expression ES-G11000 (Epson Seiko Corporation, Nagano, Japan) document scanner was turned on $30 \mathrm{~min}$ before scanning to allow it to warm up sufficiently and prevent temperature-dependent response effects. Analysis of the scanned images was performed with the DD-System (R-TECH, Inc., Tokyo, Japan). The dose profiles of all films were normalized to the central axis dose. We compared the dose profile between the $90 \%$ dose of the dose profile for DTT and the stationary phantom to evaluate geometric accuracy. ${ }^{13}$

The differences between detected and predicted positions of the iron markers were defined as the 4D modeling error. The mean $(\mu)$ and standard deviation (SD) of the absolute 4D modeling function error were analyzed. The $95^{\text {th }}$ percentile of the $4 \mathrm{D}$ modeling error $\left(4 \mathrm{D}-\mathrm{E}_{95 \%}\right)$ between the detected and predicted target position $(\mu+2 S D)$ was calculated to compare each $4 \mathrm{D}$ modeling error.

\section{Results}

\subsection{Chamber measurement}

The chamber results summarized in Table 1 indicated experimental certainty for the DTT measurements. For 1D sinusoidal pattern, the relative doses were as follows: 0.99 with non-DTT and 1.00 with DTT at $A=20$ $\mathrm{mm}$ and $T=2 \mathrm{~s} ; 0.99$ with non-DTT and 1.00 with DTT at $A=20 \mathrm{~mm}$ and $T=4 \mathrm{~s}$; and 0.84 with non-DTT and 1.00 with DTT at $A=40 \mathrm{~mm}$ and $T=4 \mathrm{~s}$. For the volunteer's respiratory pattern, the relative doses were 1.00 for non-DTT and 1.00 with DTT. The largest deviation occurred when the larger peak-to-peak amplitude was delivered with non-DTT.

Table 1: Chamber measurement results.

\begin{tabular}{cc|cc}
\hline \hline \multirow{2}{*}{$\begin{array}{c}\text { Amplitude } \\
(\mathrm{mm})\end{array}$} & $\begin{array}{c}\text { Breathing period } \\
(\mathrm{s})\end{array}$ & \multicolumn{2}{|c}{ Relative dose } \\
\cline { 3 - 4 } & 2 & non-DTT & DTT \\
20 & 4 & 0.99 & 1.00 \\
20 & 4 & 0.99 & 1.00 \\
40 & 0.84 & 1.00 \\
Volunteer's respiratory & 1.00 & 1.00 \\
\hline \hline
\end{tabular}

Chamber measurements were performed under stationary, dynamic tumor tracking (DTT), and non-DTT conditions with sinusoidal patterns (amplitude and breathing period) and a volunteer's respiratory pattern, in the cranio-caudal direction. Dose values are relative to the dose measured in the stationary phantom.

\subsection{Film measurement}

Figure 2 shows the film measurement results for the stationary phantom, DTT, and non-DTT at $A=40 \mathrm{~mm}$ and $T=4 \mathrm{~s}$. The stationary phantom and DTT had almost the same dose distribution, while non-DTT increased the blurring effects of the dose distribution. Figure 3 shows the dose profiles for the stationary phantom, DTT, and non-DTT for sinusoidal and volunteer's respiratory patterns. DTT reduced the blurring effects and produced a dose profile curve similar to that of the stationary phantom.

The film results are summarized in Table 2. Compared with the stationary conditions, the spatial distance between the $90 \%$ dose of the dose profiles were as follows: $6.53 \mathrm{~mm}$ for non-DTT and $0.40 \mathrm{~mm}$ for DTT at $A$ $=20 \mathrm{~mm}$ and $T=2 \mathrm{~s} ; 6.33 \mathrm{~mm}$ for non-DTT and $0.15 \mathrm{~mm}$ for DTT at $A=20 \mathrm{~mm}$ and $T=4 \mathrm{~s}$; and $10.61 \mathrm{~mm}$ for non-DTT and $0.17 \mathrm{~mm}$ for DTT at $A=40 \mathrm{~mm}$ and $T=4 \mathrm{~s}$. For the volunteer's respiratory pattern, the dose profiles were $6.02 \mathrm{~mm}$ (left side: $4.67 \mathrm{~mm}$, right side: $7.37 \mathrm{~mm}$ ) for non-DTT and $0.25 \mathrm{~mm}$ for DTT. The largest deviation occurred when the larger peak-to-peak amplitude was delivered with non-DTT.

Table 2: Film measurement results.

\begin{tabular}{|c|c|c|c|}
\hline \multirow{2}{*}{$\begin{array}{c}\text { Amplitude } \\
(\mathrm{mm})\end{array}$} & \multirow{2}{*}{$\begin{array}{l}\text { Breathing } \\
\text { period (s) }\end{array}$} & \multicolumn{2}{|c|}{$E_{90 \%}(\mathrm{~mm})$} \\
\hline & & non-DTT & DTT \\
\hline 20 & 2 & 6.53 & 0.40 \\
\hline 20 & 4 & 6.33 & 0.15 \\
\hline 40 & 4 & 10.61 & 0.17 \\
\hline \multicolumn{2}{|c|}{ Volunteer's respiratory } & 6.02 & 0.25 \\
\hline
\end{tabular}

Film measurements were performed under stationary, dynamic tumor tracking (DTT), and non-DTT conditions, 
with sinusoidal patterns (amplitude and breathing period) and a volunteer's respiratory pattern, in the cranio-caudal direction. Dose values are relative to the dose measured in the stationary phantom. $E_{90 \%}=$ distance of $90 \%$ dose profile between stationary and DTT or non-DTT conditions.

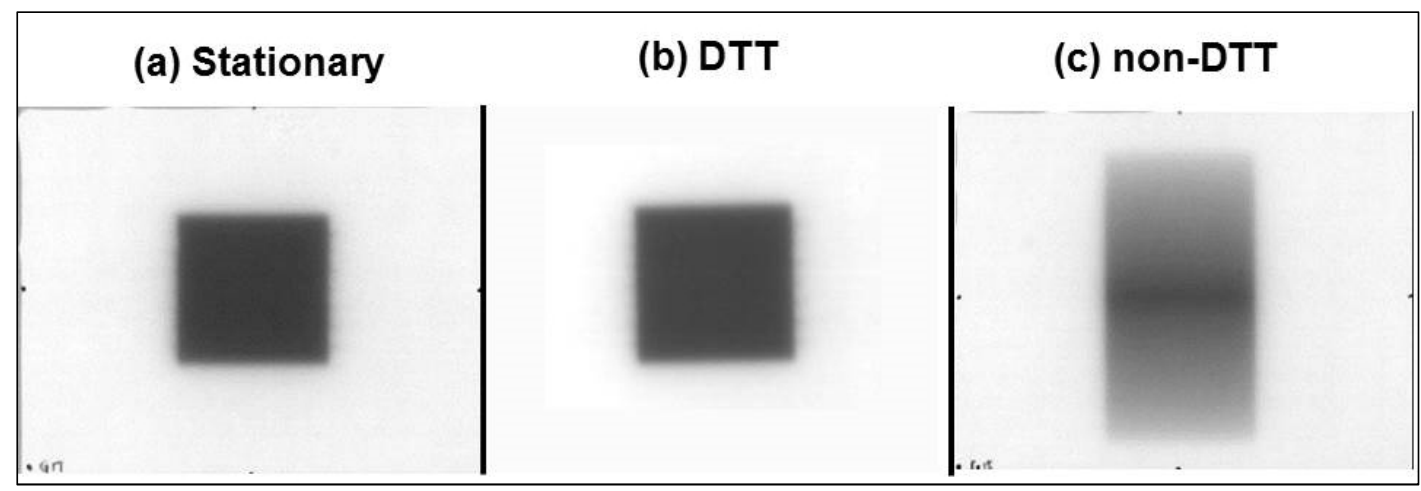

Figure 2: A single field for the one-dimensional sinusoidal pattern with an amplitude of $40 \mathrm{~mm}$ and a breathing period of $4 \mathrm{~s}$ under (a) stationary, (b) dynamic tumor tracking (DTT), and (c) non-DTT conditions. DTT clearly reduced the blurring, which was comparable to that produced by static dose distribution.

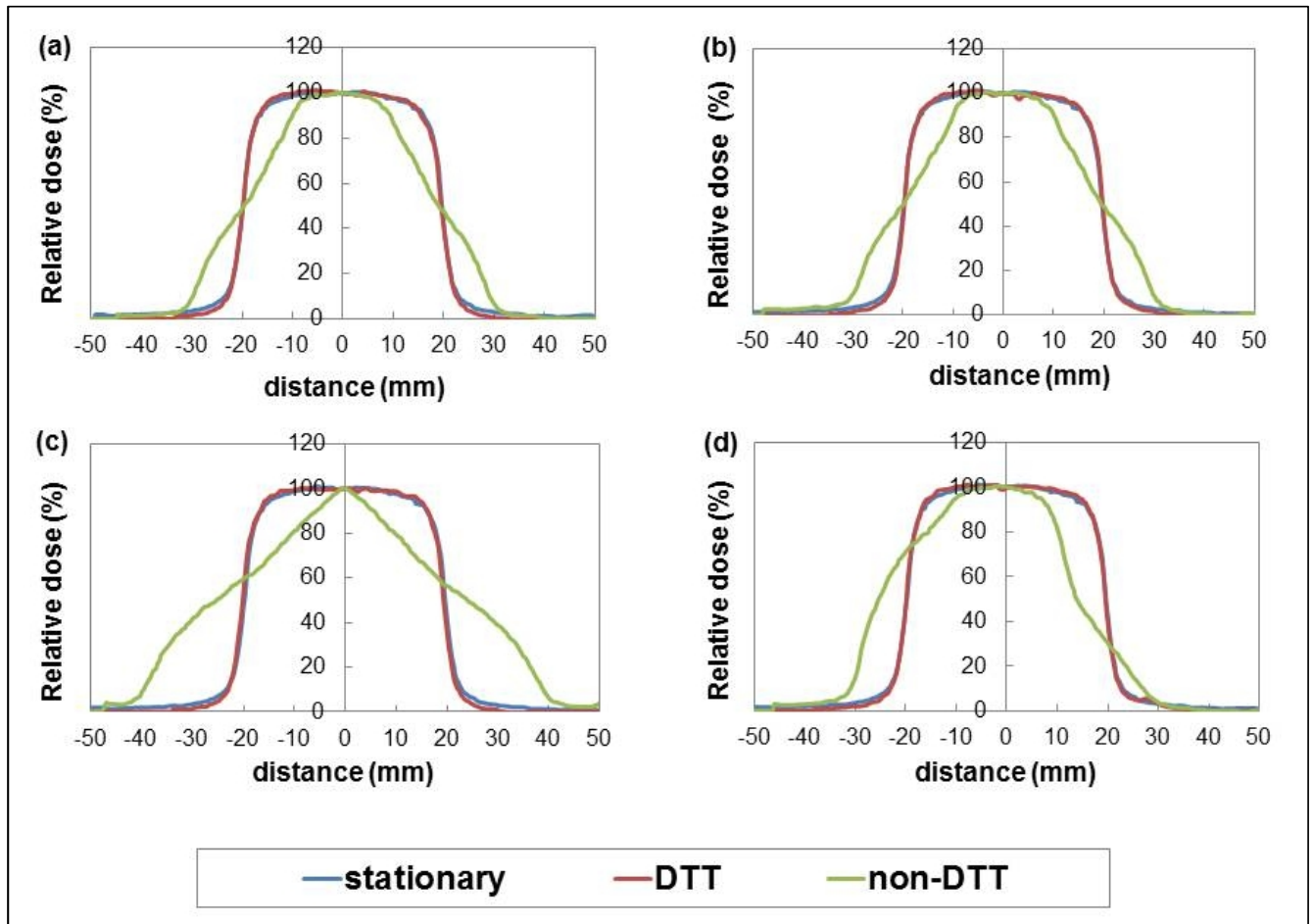

Figure 3: The dose profiles for stationary (blue line), dynamic tumor tracking (DTT; red line), and non-DTT (green line) conditions under different sinusoidal patterns (amplitude and period): (a) $20 \mathrm{~mm}, 2 \mathrm{~s}$; (b) $20 \mathrm{~mm}, 4 \mathrm{~s}$; (c) $40 \mathrm{~mm}$, $4 \mathrm{~s}$; and (d) the volunteer's respiratory pattern.

\subsection{D-modeling error}

Figure 4 shows the graph of predicted and detected target motion in the 4D modeling. As shown, the 4D-modeling error was greatest at the "end-inspiration" and "end-expiration" phases in particular (amplitude, 20 $\mathrm{mm}$; breathing period, $2 \mathrm{~s}$ ).
The 4D-modeling error results are summarized in Table 3. The largest $4 \mathrm{D}-\mathrm{E}_{95 \%}$ occurred with an amplitude of 20 $\mathrm{mm}$ and a breathing period of $2 \mathrm{~s}$. For 1D-sinusoidal patterns, the $4 \mathrm{D}-\mathrm{E}_{95 \%}$ values were $1.47 \mathrm{~mm}(A=20 \mathrm{~mm}$, $T=2 \mathrm{~s}), 0.38 \mathrm{~mm}(A=20 \mathrm{~mm}, T=4 \mathrm{~s})$, and $0.72 \mathrm{~mm} \mathrm{(} A=$ $40 \mathrm{~mm}, T=4 \mathrm{~s})$. For the volunteer's respiratory pattern, the $4 \mathrm{D}-\mathrm{E}_{95 \%}$ was $0.34 \mathrm{~mm}$. 

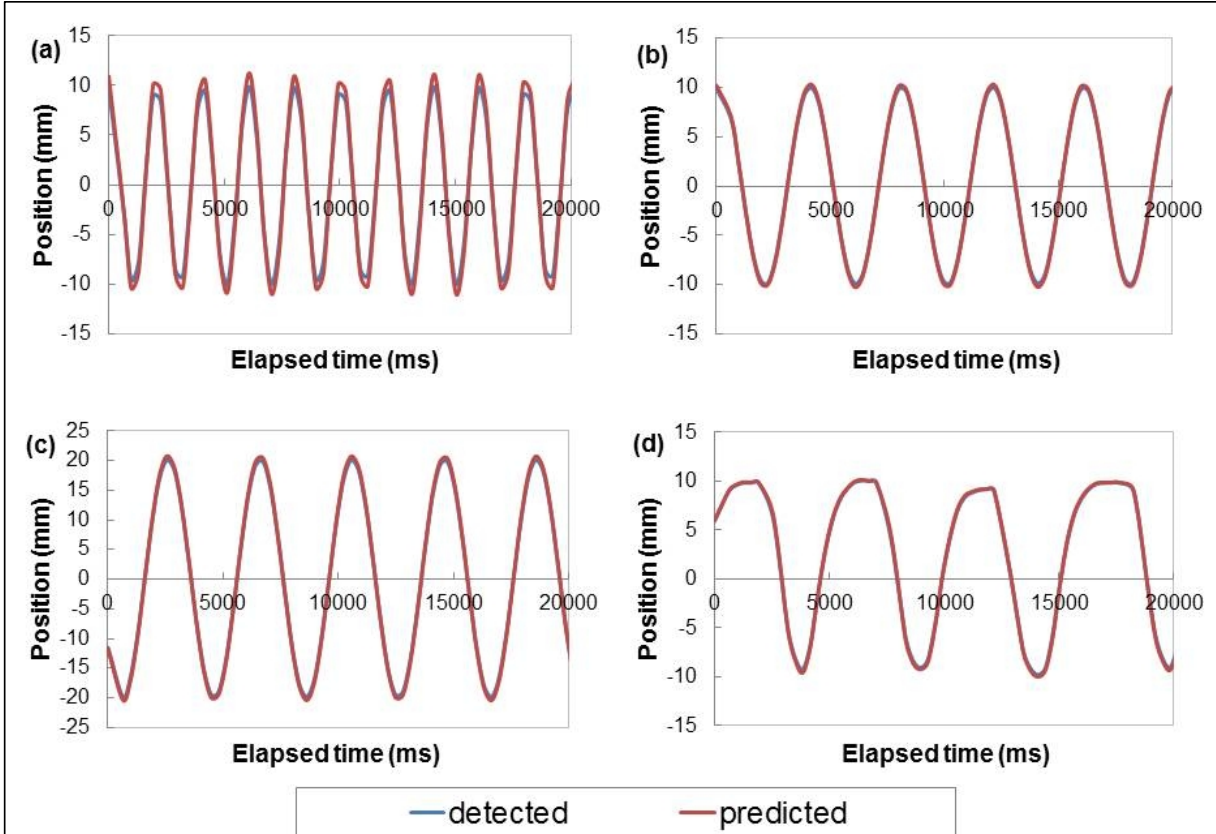

Figure 4: Graphs of detected (blue line) and predicted (red line) target motions for different sinusoidal patterns (amplitude and breathing period): (a) $20 \mathrm{~mm}, 2 \mathrm{~s}$; (b) $20 \mathrm{~mm}, 4 \mathrm{~s}$; (c) $40 \mathrm{~mm}, 4 \mathrm{~s}$; and (d) the volunteer's respiratory pattern.

\subsection{Comparison of the 4D-modeling and film measurement data}

The results for $4 \mathrm{D}-\mathrm{E}_{95 \%}$ and film measurement with DTT of both sinusoidal and the volunteer's respiratory patterns are summarized in Table 3. A good correlation was found between the $4 \mathrm{D}-\mathrm{E}_{95 \%}$ and the film measurement results $\left(R^{2}=0.70\right)$.

Table 3: The $95^{\text {th }}$ percentile of the 4D-modeling error (4D-E95\%) and film measurement result under dynamic tumor tracking for 1D sinusoidal patterns.

\begin{tabular}{cc|cc}
\hline \hline $\begin{array}{c}\text { Amplitude } \\
(\mathrm{mm})\end{array}$ & $\begin{array}{c}\text { Breathing period } \\
(\mathrm{s})\end{array}$ & $\begin{array}{c}4 D-E_{95 \%} \\
(\mathrm{~mm})\end{array}$ & $\begin{array}{c}\text { Film result } \\
(\mathrm{mm})\end{array}$ \\
\hline 20 & 2 & 1.47 & 0.40 \\
20 & 4 & 0.38 & 0.15 \\
40 & 4 & 0.72 & 0.17 \\
Volunteer's respiratory & 0.34 & 0.25 \\
\hline \hline
\end{tabular}

Results are for 1D sinusoidal pattern (amplitude and breathing period) and a volunteer's respiratory pattern, in the cranio-caudal direction. $4 \mathrm{D}-\mathrm{E}_{95} \%=4 \mathrm{D}$ - modeling error between the detected and predicted target position $(\mu+2 \mathrm{SD})$.

\section{Discussion}

The AAPM-TG142 recommends the examination of respiratory-gated accelerator operation, measurement of beam energy and output constancy, and measurement of the temporal accuracy of phase/amplitude gating windows; but it provides no details regarding the performance of these examinations. ${ }^{10}$ Recently, woods et al. reported detailed and comprehensive guidance for respiratory gating commissioning and routine $\mathrm{QA}$ in accordance with AAPM - TG142. ${ }^{14}$ As for real-time tumor-tracking radiotherapy systems, Shiinoki et al. suggested a QA procedure for respiratory-gated radiation that reduced the blurring effects on dose distribution with high dosimetric and geometric accuracy..$^{13}$ However, there are no existing procedural guidelines specifically for DTT. It is important that the accuracy of DTT be measured prior to treatment.

In this study, the gimbals head was swung to a maximum angle of 1.2 degrees, which produced a distance between the target and radiation source of $100.02 \mathrm{~cm}$. At this angle, the beam path hardly changed from that at the zero-degree angle. Therefore, the relative dose was 1.00 for DTT in a single $40 \times 40 \mathrm{~mm}$ field. For non-DTT, motion caused blurring of the dose profile that caused an increased beam penumbra. In contrast, DTT dramatically reduced the blurring dose profile and produced a penumbra that was similar to that of the stationary phantom. The volunteer's respiratory pattern reflected the motion probability density function. ${ }^{15}$

The 4D-modeling error was increased on the target motion amplitude, and the peak position sometimes overestimated the predicted position. Largest 4D-E95\% was occurred under rapidly breathing pattern, because X-ray imaging with 320 or $640 \mathrm{~ms}$ cannot detect iron markers at the "end-inspiration" and "end-expiration" phases. Thereby, slight blurring was generated on the $90 \%$ dose profile. However, the 4D-modeling error may be predicted by mechanical errors in DTT. Mukumoto et al. reported that positional tracking errors correlated strongly with 4D-modeling errors, which resulted from miscorrelations between target and IR marker motions. ${ }^{6}$ 
In the moving phantom study, the correlation between 4D-modeling and film dosimetric errors was very small because the motions of the target and the infrared markers were perfectly synchronized. The sinusoidal pattern is easily reproducible and predictable.

A limitation of this study was that it was performed under 1D sinusoidal regulated moving target in the CC direction only. Further studies should examine the correlation between 4D modeling and tracking accuracy under various conditions and in different directions.

\section{Conclusion}

Our present results indicate the high dosimetric and geometric accuracy of DTT when using 4D modeling and that its use dramatically reduced the blurring effects on dose distribution when compared with those with non-DTT. We recommend the use of a QA procedure for DTT performed with the Vero4DRT ${ }^{\mathrm{TM}}$ system.

\section{Conflict of interest}

The authors declare that they have no conflicts of interest. The authors alone are responsible for the content and writing of the paper.

\section{References}

1. Matsuo Y, Ueki N, Takayama K, et al. Evaluation of dynamic tumour tracking radiotherapy with real-time monitoring for lung tumours using a gimbal mounted linac. Radiother Oncol. 2014; 112:360-4.

2. Inoue $\mathrm{T}$, Katoh $\mathrm{N}$, Onimaru, et al. Stereotactic body radiotherapy using gated radiotherapy with real-time tumor-tracking for stage I non-small cell lung cancer. Radiat Oncol. 2013; 69:1186.

3. Nagata Y, Wulf J, Lax I, et al. Stereotactic radiotherapy of primary lung cancer and other targets: results of consultant meeting of the International Atomic Energy Agency. Int J Radiat Oncol Biol Phys. 2011;79:660-9.

4. Rosenzweig KE, Hanley J, Mah D, et al. The deep inspiration breath-hold technique in the treatment of inoperable non- small-cell lung cancer. Int J Radiat Oncol Biol Phys. 2000; 48:81-7.

5. Shirato H, Shimizu S, Kunieda T, et al. Physical aspects of a real-time tumor-tracking system for gated radiotherapy. Int J Radiat Oncol Biol Phys. 2000;48:1187-95.

6. Mukumoto N, Nakamura M, Sawada A, et al. Accuracy verification of infrared marker-based dynamic tumor-tracking irradiation using the gimbaled x-ray head of the Vero4DRT (MHI-TM2000). Med Phys. 2013;40:041706.

7. Takayama K, Mizowaki T, Kokubo M, et al. Initial validations for pursuing irradiation using a gimbals tracking system. Radiother Oncol. 2009;93:45-9.

8. Kamino Y, Takayama K, Kokubo M, et al. Development of a four-dimensional image-guided radiotherapy system with a gimbaled X-ray head. Int J Radiat Oncol Biol Phys. 2006;66:271-8.

9. Keall PJ, Mageras GS, Balter JM, et al. The management of respiratory motion in radiation oncology report of AAPM Task Group 76. Med Phys. 2006;33:3874-900.

10. Klein EE, Hanley J, Bayouth J, et al. Task Group 142 Report: Quality assurance of medical accelerators. Med Phys. 2009;36:4197-242.

11. Akimoto $M$, Nakamura $M$, Mukumoto $N$, et al. Predictive uncertainty in infrared marker-based dynamic tumor tracking with Vero4DRT. Med Phys. 2013; 40: 091705.

12. Nakamura $M$, Akimoto $M$, Mukumoto $N$, et al. Influence of the correlation modeling period on the prediction accuracy of infrared marker-based dynamic tumor tracking using a gimbaled X-ray head. Phys Med. 2015;31:204-9.

13. Shiinoki T, Kawamura S, Uehara T, et al. Quality assurance for respiratory-gated radiotherapy using the real-time tumor-tracking radiotherapy system. International Journal of Medical Physics, Clinical Engineering and Radiation Oncology. 2014;3:125-32.

14. Woods H, Rong Y. TG-142 compliant and comprehensive quality assurance tests for respiratory gating. Med Phys. 2015;42:6488.

15. Bortfeld T, Jiang SB, Rietzei E. Effects of motion on the total dose distribution. Semin Radiat Oncol. 2004;14:41-51. 